\title{
ON THE CONVEXITY OF THE VALUE FUNCTION FOR A CLASS OF NONCONVEX VARIATIONAL PROBLEMS: EXISTENCE AND OPTIMALITY CONDITIONS*
}

\author{
F. FLORES-BAZÁN ${ }^{\dagger}$, A. JOURANI ${ }^{\ddagger}$, AND G. MASTROENI ${ }^{\S}$
}

\begin{abstract}
In this paper we study a class of perturbed constrained nonconvex variational problems depending on either time/state or time/state's derivative variables. Its (optimal) value function is proved to be convex and then several related properties are obtained. Existence, strong duality results, and necessary/sufficient optimality conditions are established. Moreover, via a necessary optimality condition in terms of Mordukhovich's normal cone, it is shown that local minima are global. Such results are given in terms of the Hamiltonian function. Finally various examples are exhibited showing the wide applicability of our main results.
\end{abstract}

Key words. nonconvex variational problems, Lyapunov theorem, existence of minima, Hamiltonian, strong duality, local minima

AMS subject classifications. 49J15, 49J52, 49K15, 49N15, 90C46

DOI. $10.1137 / 14096877 \mathrm{X}$

1. Introduction and formulation of the problem. We consider, given $a \in$ $\mathbb{R}^{m}$, the following class of minimization problems $(P(a))$ :

$$
\inf \left\{\int_{0}^{1} f_{0}(t, z(t)) d t: z \in K(a)\right\},
$$

where

$$
K(a) \doteq\left\{z \in L^{1}\left([0,1], \mathbb{R}^{n}\right): z \in \mathcal{Z}, \int_{0}^{1} g_{0}(t, z(t)) d t \in-W+a\right\} .
$$

Here, $W \subseteq \mathbb{R}^{m}$ is nonempty, closed, and convex; $\mathcal{Z}$ is the set of functions $z \in$ $L^{1}\left([0,1], \mathbb{R}^{n}\right)$ satisfying $z(t) \in Z(t)$ for a.e. $t \in[0,1]$, with $Z:[0,1] \rightrightarrows \mathbb{R}^{n}$ a measurable set-valued mapping with nonempty and closed values; $f_{0}:[0,1] \times \mathbb{R}^{n} \rightarrow \mathbb{R} \cup\{+\infty\}$, $g_{0}:[0,1] \times \mathbb{R}^{n} \rightarrow \mathbb{R}^{m}$, and $f_{0}(t, \cdot)$ is lower semicontinuous (lsc) and $g_{0}(t, \cdot)$ is continuous for a.e. $t \in[0,1] ; f_{0}$ is a Borel function and $g_{0}(\cdot, z)$ is measurable (with respect to Lebesgue measure) for all $z \in \mathbb{R}^{n}$ such that $g_{0}(\cdot, z(\cdot)) \in L^{1}\left([0,1], \mathbb{R}^{m}\right)$ for all $z \in \mathcal{Z}$. We consider the functions $f: L^{1}\left([0,1], \mathbb{R}^{n}\right) \rightarrow \mathbb{R} \cup\{+\infty\}$ and $g: L^{1}\left([0,1], \mathbb{R}^{n}\right) \rightarrow \mathbb{R}^{m}$ defined by

$$
f(z) \doteq \int_{0}^{1} f_{0}(t, z(t)) d t, \quad g(z) \doteq \int_{0}^{1} g_{0}(t, z(t)) d t .
$$

Furthermore, we impose the following assumptions on $f_{0}$ :

*Received by the editors May 12, 2014; accepted for publication (in revised form) September 4, 2014; published electronically November 13, 2014. The first two authors thank Math-AmSud 13math01 and the ECOS project for their support.

http://www.siam.org/journals/sicon/52-6/96877.html

${ }^{\dagger}$ Departamento de Ingeniería Matemática and $\mathrm{CI}^{2} \mathrm{MA}$, Universidad de Concepción, Casilla 160C, Concepción, Chile (fflores@ing-mat.udec.cl). This author's research was supported in part by CONICYT-Chile through FONDECYT 112-0980 and BASAL projects, CMM, Universidad de Chile.

‡Université de Bourgogne, Institut de Mathématiques de Bourgogne, UMR 5584 CNRS, BP 47 870, 21078 Dijon Cedex, France (jourani@u-bourgogne.fr).

$\S$ Department of Computer Science, University of Pisa, L.go B. Pontecorvo 3, 56100 Pisa, Italy (gmastroeni@di.unipi.it). 
- there exists $z_{0} \in \mathcal{Z}$ such that

$$
\int_{0}^{1} f_{0}\left(t, z_{0}(t)\right) d t<+\infty
$$

- there exist $\alpha \in \mathbb{R}^{n}$ and $\beta \in L^{1}([0,1], \mathbb{R})$ satisfying

$$
f_{0}(t, z) \geq\langle\alpha, z\rangle+\beta(t) \text { for a.e. } t \in[0,1] \text { for all } z \in \mathbb{R}^{n} .
$$

Under the latter assumption, $f(z)>-\infty$ for all $z \in L^{1}\left([0,1], \mathbb{R}^{n}\right)$.

Problems like (1.1) subsume an important class of variational problems, namely,

$$
\inf \left\{\int_{0}^{1} f_{0}(t, \dot{x}(t)) d t: x \in W^{1,1}\left([0,1], \mathbb{R}^{n}\right): x(0)=a, x(1)=b\right\},
$$

where $W^{1,1}\left([0,1], \mathbb{R}^{n}\right)$ denotes the space of absolutely continuous functions from $[0,1]$ to $\mathbb{R}^{n}$, and $a, b$ are given vectors in $\mathbb{R}^{n}$.

Several standard models in mathematical economics can be written in the form of (1.1) with $W=\mathbb{R}_{+}^{n}$; see [1] for instance. One of the economic interpretations deals with the investigation of markets with a continuum of traders in an economy with production, in which case, $z_{j}(t)$ means the amount of the commodity $j$ to be bought (produced) by the trader $t$. Thus, the integral in (1.1) gives the total expenditure (cost) of the overall coalition under the commodity assignment $z$. The integral appearing in (1.2) has obvious meaning. A model related to a continuous-time allocation process for a continuum of traders with unilateral constraints may be found in [7].

The classical existence result due to Tonelli requires the convexity and superlinear growth assumptions on $f_{0}(t, \cdot)$, which imply the weak lower semicontinuity of the integral functional and the weak compactness of its sublevel sets (see, for instance, Theorem 16.2 in [5]). Our goal is to avoid such assumptions by analyzing carefully the value function associated with (1.1) depending on $a$, which allows us to consider integrands with slow growth. Important existence results, including the nonoccurrence of the Lavrentiev phenomenon, recently obtained for nonconvex optimal control and variational problems, but using different approaches, can be found in [17]. In particular, generic results, in the sense of Baire category, were also presented.

The particular case $g_{0}(t, z)=z$ and $W=\{0\}$ was considered in [6], and under convexity of $f_{0}(t, \cdot)$ in $[9]$.

One of the main goals of the present paper, after a careful analysis of the value function, is to provide a necessary and sufficient optimality condition of zero order for a feasible solution to problem $(P(0))$ to be optimal (Corollary 3.7), along with sufficient and/or necessary conditions for the same problem via the Hamiltonian.

The structure of the paper is as follows. Section 2 deals with some basic notation, definitions, and preliminaries; in particular, a variant of the Lyapunov convexity theorem (Theorem 2.1), suitable for our purpose, is recalled. Section 3 describes the most important properties of the value function (including convexity) to be used in subsequent sections. In section 4 , we establish necessary optimality conditions via the subdifferential of the optimal value function. Some of the results of the preceding section are applied in section 5 to prove that local minimality implies global minimality for the problem (1.1). In connection with assumption (5.1), section 6 provides several equivalent conditions implying the previous assumption. Finally section 7 establishes some formulas for computing the value function via the Hamiltonian, and some existence results as well. 
2. Basic notation and preliminaries. Given two vectors $a, b$ in $\mathbb{R}^{n},\langle a, b\rangle$ denotes their inner or scalar product. A set $P \subseteq \mathbb{R}^{n}$ is said to be a cone if $t P \subseteq P$ for all $t \geq 0$. For a given $A \subseteq \mathbb{R}^{n}: \bar{A}, \operatorname{co}(A)$, int $A$, bd $A$, stand for the closure of $A$, the convex hull of $A$ (the smallest convex set containing $A$ ), topological interior of $A$, boundary of $A$, respectively. Furthermore cone $(A)$ denotes the smallest cone containing $A$, that is,

$$
\operatorname{cone}(A)=\bigcup_{t \geq 0} t A
$$

$\underline{\text { whereas }} \overline{\operatorname{cone}}(A)$ denotes the smallest closed cone containing $A$ : obviously $\overline{\operatorname{cone}}(A)=$ $\overline{\operatorname{cone}(A)}$.

Moreover, $x \in A$ is said to be a relative interior point of $A$ if $\overline{\operatorname{cone}}(A-x)$ is a vector space (see, for instance, [2]). The set of relative interior points of $A$ is denoted by ri $A$. It is well known that in the case $A$ is convex, $x \in \operatorname{ri} A$ if and only if $x$ is an interior point with respect to the affine hull of $A$ or, equivalently, if $N(A ; x)$ is a vector space, where $N(A ; x) \doteq\left\{\xi \in \mathbb{R}^{n}:\langle\xi, a-x\rangle \leq 0, \quad \forall a \in A\right\}$, is the (outward) normal cone to $A$ at $x \in A$.

The positive polar of the convex cone $P \subseteq \mathbb{R}^{n}$ is defined by

$$
P^{*} \doteq\left\{y^{*} \in \mathbb{R}^{n}:\left\langle y^{*}, x\right\rangle \geq 0, \forall x \in P\right\} .
$$

We now recall an extension of the Lyapunov theorem proved in [13]. This plays an important role in the existence theory for optimal control problems without convexity assumptions; see, for instance, [6] and references therein.

Given a set $K \subseteq L^{1}\left([0,1], \mathbb{R}^{k}\right)$, define the set

$$
I(K) \doteq\left\{\int_{0}^{1} \phi(t) d t: \phi \in K\right\} .
$$

$K$ is said to be decomposable if, for every measurable set $B \subseteq[0,1]$ and all $u, v \in K$,

$$
u \cdot \chi_{B}+v \cdot \chi_{[0,1] \backslash B} \in K,
$$

where $\chi_{B}$ is the characteristic function of the set $B$, i.e., $\chi_{B}(x)=1$ if $x \in B$, and $\chi_{B}(x)=0$ elsewhere.

The next theorem is taken from [13] and provides a simple existence result for problem (1.1) as a consequence of Corollary 3.3, as Remark 3.4 shows.

Theorem 2.1 (see $[13$, Theorem 4$]$ ). If $K \subseteq L^{1}\left([0,1], \mathbb{R}^{k}\right)$ is decomposable, then $I(K)$ is convex and $I(K)=I($ co $K)$. If, in addition, $K$ is (strong) closed and the closure of $I(K)$ contains neither a line nor an extremal half-line, then $I(K)$ is closed.

In what follows, we recall some main facts about envelopes of functions. Given $h: \mathbb{R}^{n} \rightarrow \mathbb{R} \cup\{ \pm \infty\}, \bar{h}, \overline{c o} h$ stand for the greatest lsc function not larger than $h$ and for the greatest convex and lsc function not larger than $h$, respectively. To be coherent with our previous notation we need the following definition of epigraph of a function:

$$
\text { epi } h \doteq\left\{(t, x) \in \mathbb{R} \times \mathbb{R}^{n}: h(x) \leq t\right\}
$$

and

$$
\operatorname{epi}^{0} h \doteq\left\{(t, x) \in \mathbb{R} \times \mathbb{R}^{n}: h(x)<t\right\} .
$$

Copyright $\odot$ by SIAM. Unauthorized reproduction of this article is prohibited. 
In the case $h$ is convex, we have (see [16, Lemma 7.3])

$$
\operatorname{ri}(\text { epi } h)=\left\{(t, x) \in \mathbb{R} \times \mathbb{R}^{n}: x \in \operatorname{ri}(\operatorname{dom} h), h(x)<t\right\} .
$$

It is known that

$$
\text { epi } \bar{h}=\overline{\text { epi } h} ; \quad \overline{\operatorname{co}}(\text { epi } h)=\text { epi } \overline{\operatorname{co}} h .
$$

Moreover, if $\overline{\mathrm{co}} h(x)>-\infty$ for all $x \in \mathbb{R}^{n}$, then $\overline{\mathrm{co}} h(x)=h^{* *}(x)$ for all $x \in \mathbb{R}^{n}$, where $h^{* *} \doteq\left(h^{*}\right)^{*}$ is the bipolar or biconjugate of $h$, that is, the conjugate of $h^{*}$. There are examples showing the assumption $\overline{\text { co }} h(x)>-\infty$ for all $x \in \mathbb{R}^{n}$ is necessary to get the previous equality. In general we have $h^{* *} \leq \overline{\mathrm{co}} h \leq h$.

For any nonempty set $W \subseteq \mathbb{R}^{m}$, and $a, b \in \mathbb{R}^{m}$, we write $a \leq_{W} b$ (or equivalently, $\left.b \geq_{W} a\right)$ if $b-a \in W$.

The indicator function $\iota_{S}$ of the set $S$ is defined by $\iota_{S}(x)=0$ if $x \in S$ and $+\infty$ otherwise.

The space of absolutely continuous functions from $[0,1]$ into $\mathbb{R}^{k}$ is denoted by $W^{1,1}\left([0,1], \mathbb{R}^{k}\right)$, and it is equipped with the norm

$$
\|x\|_{1,1}=\|x(0)\|+\int_{0}^{1}\|\dot{x}(t)\| d t .
$$

It is well known that $W^{1,1}\left([0,1], \mathbb{R}^{k}\right)$ is a Banach space. Set $W_{k}^{1,1} \doteq W^{1,1}\left([0,1], \mathbb{R}^{k}\right)$ and $L^{1} \doteq L^{1}\left([0,1], \mathbb{R}^{n}\right)$. As usual, the norm on the product space $W_{k}^{1,1} \times L^{1}$ is the sum of the norms of $W_{k}^{1,1}$ and $L^{1}$, and it will be denoted by $\|\cdot\|$.

Set $\mathbb{R}_{+} \doteq\left[0,+\infty\left[, \mathbb{R}_{++} \doteq\right] 0,+\infty\left[, \mathbb{R}_{--}=-\mathbb{R}_{++}\right.\right.$.

In what follows we use the convention $+\infty-(+\infty)=+\infty$.

3. The convexity of the optimal value function and related properties. We associate with problem (1.1) the optimal value function $\psi: \mathbb{R}^{m} \rightarrow \mathbb{R} \cup\{ \pm \infty\}$ defined as follows:

$$
\psi(a)=\left\{\begin{array}{c}
\inf \left\{\int_{0}^{1} f_{0}(t, z(t)) d t: \quad g(z) \in-W+a, \quad z \in \mathcal{Z}\right\} \text { if } K(a) \neq \emptyset \\
+\infty \quad \text { otherwise. }
\end{array}\right.
$$

Consider the Lagrangian $L(\lambda, z)=f(z)+\langle\lambda, g(z)\rangle, \lambda \in W^{*}, z \in L^{1}\left([0,1], \mathbb{R}^{n}\right)$, and define the dual of problem $(P(0))(a=0$ in (1.1)) by

$$
v_{D} \doteq \sup _{\lambda \in W^{*}} \inf _{z \in \mathcal{Z}} L(\lambda, z)
$$

We consider a classic result (see, e.g., [15, Theorem 7]), although proved under convexity conditions, which relates the optimal value $v_{D}$ of (3.1) with the biconjugate of the value function $\psi$.

TheOrem 3.1. Assume that $f, g$ are defined as in (1.3), with $W$ being additionally a cone, and $K(0) \neq \emptyset$. Then $v_{D}=\psi^{* *}(0)$.

Proof. Define $F: \mathcal{Z} \times \mathbb{R}^{m} \longrightarrow \mathbb{R} \cup\{ \pm \infty\}$ :

$$
F(z, a)= \begin{cases}f(z) & \text { if } g(z) \in-W+a \\ +\infty & \text { otherwise }\end{cases}
$$

Copyright $@$ by SIAM. Unauthorized reproduction of this article is prohibited. 
Then the Lagrangian function can be written as

$$
L(\lambda, z)=\inf _{a \in \mathbb{R}^{m}}\{F(z, a)+\langle\lambda, a\rangle\}=\left\{\begin{array}{l}
f(z)+\langle\lambda, g(z)\rangle \text { if } \lambda \in W^{*}, \\
-\infty \text { if } \lambda \notin W^{*}, f(z)<+\infty, \\
+\infty \text { if } \lambda \notin W^{*}, \quad f(z)=+\infty,
\end{array}\right.
$$

and the value function as

$$
\psi(a)=\inf _{z \in \mathcal{Z}} F(z, a)
$$

Therefore

$$
\inf _{z \in \mathcal{Z}} L(\lambda, z)=\inf _{a \in \mathbb{R}^{m}}\left\{\inf _{z \in \mathcal{Z}} F(z, a)+\langle\lambda, a\rangle\right\}=-\sup _{a \in \mathbb{R}^{m}}\{\langle-\lambda, a\rangle-\psi(a)\}=-\psi^{*}(-\lambda) .
$$

Then,

$$
\sup _{\lambda \in W^{*}} \inf _{z \in \mathcal{Z}} L(\lambda, z)=\sup _{\lambda \in \mathbb{R}^{m}} \inf _{z \in \mathcal{Z}} L(\lambda, z)=\sup _{\lambda \in \mathbb{R}^{m}}\left[-\psi^{*}(-\lambda)\right]=\psi^{* *}(0) .
$$

Set $C_{0} \doteq \operatorname{dom} f \cap \mathcal{Z}=\{z \in \mathcal{Z}: f(z)<+\infty\}$ and

$$
\begin{aligned}
K_{0} \doteq\left\{(u, v) \in L^{1}\left([0,1], \mathbb{R}^{1+m}\right):\right. & \exists z \in \mathcal{Z}, u(t) \geq f_{0}(t, z(t)), \\
& \left.v(t) \geq_{W} g_{0}(t, z(t)), \text { for a.e. } t \in[0,1]\right\} .
\end{aligned}
$$

We get the following result which is important by itself.

Theorem 3.2. Let $F(z) \doteq(f(z), g(z))$ with $f, g$ defined as in (1.3) and $W \subseteq \mathbb{R}^{m}$ being any nonempty closed and convex set. The following assertions hold:

(a) The set $K_{0}$ is decomposable, $I\left(K_{0}\right)$ is convex, and $I\left(K_{0}\right)=F\left(C_{0}\right)+$ $\left(\mathbb{R}_{+} \times W\right) \subseteq$ epi $\psi$.

(b) $(r, a) \in$ epi $\psi \Longleftrightarrow\left(r+\frac{1}{k}, a\right) \in F\left(C_{0}\right)+\left(\mathbb{R}_{+} \times W\right) \quad \forall k \in \mathbb{N}$.

Consequently, the function $\psi: \mathbb{R}^{m} \rightarrow \mathbb{R} \cup\{ \pm \infty\}$ is convex, and

$$
I\left(K_{0}\right) \subseteq \text { epi } \psi \subseteq \overline{I\left(K_{0}\right)} .
$$

(c) We have

$$
\begin{aligned}
& (r, a) \in \operatorname{ri}(\operatorname{epi} \bar{\psi}) \Longleftrightarrow a \in \operatorname{ri}(\operatorname{dom} \bar{\psi}) \\
& \quad \text { and } \exists k_{0} \in \mathbb{N}, \quad\left(r-\frac{1}{k}, a\right) \in \operatorname{epi} \psi \quad \forall k \geq k_{0} .
\end{aligned}
$$

As a consequence, if $A \subseteq \mathrm{ri}(\operatorname{dom} \bar{\psi})$ then,

$$
\begin{aligned}
(\text { ri epi } \bar{\psi}) \cap(\mathbb{R} \times A) & =\operatorname{epi}^{0} \psi \cap(\mathbb{R} \times A), \\
\text { epi } \bar{\psi} \cap(\mathbb{R} \times A) & =\text { epi } \psi \cap(\mathbb{R} \times A) .
\end{aligned}
$$

Proof.

(a) We observe first that $K_{0}$ is a decomposable set. Indeed, let $\left(u_{i}, v_{i}\right) \in K_{0}$, $i=1,2$, and $B \subseteq[0,1]$ a measurable set. Then, for some $z_{i} \in L^{1}, i=1,2$, we have

$$
u_{i}(t) \geq f_{0}\left(t, z_{i}(t)\right), \quad v_{i}(t) \geq_{W} g_{0}\left(t, z_{i}(t)\right), \quad z_{i}(t) \in Z(t) \text { for a.e. } t \in[0,1] .
$$

Copyright (c) by SIAM. Unauthorized reproduction of this article is prohibited. 
Clearly $z_{i} \in C_{0}$ for $i=1,2$. Setting $\tilde{z} \doteq z_{1} \cdot \chi_{B}+z_{2} \cdot \chi_{[0,1] \backslash B} \in L^{1}$, we have for a.e. $t \in[0,1]: \tilde{z}(t) \in Z(t)$ and

$$
\begin{aligned}
u_{1}(t) \cdot \chi_{B}(t)+u_{2}(t) \cdot \chi_{[0,1] \backslash B}(t) & \geq f_{0}(t, \tilde{z}(t)), \\
v_{1}(t) \cdot \chi_{B}(t)+v_{2}(t) \cdot \chi_{[0,1] \backslash B}(t) & \geq{ }_{W} g_{0}(t, \tilde{z}(t)),
\end{aligned}
$$

i.e., $\left(u_{1}, v_{1}\right) \cdot \chi_{B}+\left(u_{2}, v_{2}\right) \cdot \chi_{[0,1] \backslash B} \in K_{0}$, proving the decomposability of $K_{0}$. Thus the convexity of $I\left(K_{0}\right)$ follows from Theorem 2.1.

To prove the equality $I\left(K_{0}\right)=F\left(C_{0}\right)+\left(\mathbb{R}_{+} \times W\right)$, first notice that $I\left(K_{0}\right) \subseteq$ $F\left(C_{0}\right)+\left(\mathbb{R}_{+} \times W\right)$ is straightforward by the convexity and closedness of $W$. For the reverse inclusion it is enough to observe that if $(u, v) \in F\left(C_{0}\right)+$ $\left(\mathbb{R}_{+} \times W\right)$, then, for some $z \in C_{0}$ and $(h, p) \in\left(\mathbb{R}_{+} \times W\right)$,

$$
(u, v)=\left(\int_{0}^{1}\left[f_{0}(t, z(t))+h\right] d t, \int_{0}^{1}\left[g_{0}(t, z(t))+p\right] d t\right) \in I\left(K_{0}\right),
$$

proving the equality in $(a)$. This also shows that $F\left(C_{0}\right)+\left(\mathbb{R}_{+} \times W\right) \subseteq$ epi $\psi$. (b) By taking into account the inclusion in $(a)$, the "only if" part is easily obtained. Let $\psi(a) \leq r<+\infty$. Then $K(a) \neq \emptyset$, and for all $k \in \mathbb{N}$ there exists $z_{k} \in C_{0}$ such that $f\left(z_{k}\right)<r+\frac{1}{k}$ and $g\left(z_{k}\right) \leq_{W} a$. Thus

$$
\left(r+\frac{1}{k}, a\right)=\left(f\left(z_{k}\right), g\left(z_{k}\right)\right)+\left(r+\frac{1}{k}-f\left(z_{k}\right), a-g\left(z_{k}\right)\right) \in F\left(C_{0}\right)+\left(\mathbb{R}_{+} \times W\right),
$$

which completes the proof of the equivalence.

The convexity of $\psi$ follows from $(a)$ which asserts the convexity of $I\left(K_{0}\right)=$ $F\left(C_{0}\right)+\left(\mathbb{R}_{+} \times W\right)$.

Combining $(a)$ and the last equivalence, we get (3.4).

(c) Let $(r, a) \in \operatorname{ri}($ epi $\bar{\psi})$. By $(2.1), a \in \operatorname{ri}(\operatorname{dom} \psi)$ and $\bar{\psi}(a)<r$. For $k_{0} \in \mathbb{N}$ sufficiently large, we have $\bar{\psi}(a)<r-\frac{1}{k}<r$ for all $k \geq k_{0}$. Thus, for such $k \in \mathbb{N}$, one obtains

$$
\left(r-\frac{1}{k}, a\right) \in \operatorname{ri}(\text { epi } \bar{\psi})=\operatorname{ri} \overline{I\left(K_{0}\right)}=\operatorname{ri} I\left(K_{0}\right) .
$$

Moreover, by convexity again, ri (epi $\psi)=\operatorname{ri}(\overline{\operatorname{epi} \psi})=\operatorname{ri}($ epi $\bar{\psi})$, proving one implication of the equivalence. The other is trivial.

One inclusion in (3.5) follows from the previous equivalence and the other is straightforward.

For (3.6) we need to check the inclusion " $\subseteq$." Let us take any $(r, a) \in$ epi $\bar{\psi}$ with $a \in A$. Then, we have two possibilities: $\bar{\psi}(a)<r$ or $\bar{\psi}(a)=r$. In the first case, we get $(r, a) \in \operatorname{ri}(\operatorname{epi} \bar{\psi})$ and so $(r, a) \in \operatorname{epi} \psi$. In the second case, since $\bar{\psi}(a)=r<r+\frac{1}{k}$, we obtain $\left(r+\frac{1}{k}, a\right) \in \operatorname{ri}(\operatorname{epi} \bar{\psi})$. By $(3.5), \psi(a)<r+\frac{1}{k}$ for all $k \in \mathbb{N}$, and the conclusion follows.

It is not difficult to check that

$$
\operatorname{dom} \psi=g\left(C_{0}\right)+W \text {. }
$$

Thus, since $F\left(C_{0}\right)+\left(\mathbb{R}_{+} \times W\right)$ is convex, we obtain immediately the convexity of $g\left(C_{0}\right)+W$, i.e., dom $\psi$ is convex, which is also a consequence of the convexity of $\psi$. This along with other results, which follow from (3.6), are summarized in the following corollary. 
COROLlary 3.3. Under the above assumptions, the following hold:

(a) $\operatorname{ri}(\operatorname{dom} \bar{\psi})=\operatorname{ri}(\operatorname{dom} \psi), \overline{\operatorname{dom} \psi}=\overline{\operatorname{dom} \bar{\psi}}$, and $\bar{\psi}(a)=\psi(a) \forall a \in \operatorname{ri}(\operatorname{dom} \bar{\psi})$. Consequently,

$$
\operatorname{ri}(\operatorname{epi} \bar{\psi})=\operatorname{ri}(\operatorname{epi} \psi)=\{(r, a) \in \mathbb{R} \times \operatorname{ri}(\operatorname{dom} \psi): \psi(a)<r\} .
$$

(b) For $a \in \operatorname{ri}(\operatorname{dom} \psi)$ with $\psi(a) \in \mathbb{R}$, one has

$$
\begin{aligned}
\psi(a) & =\min \left\{r \in \mathbb{R}: \quad(r, a) \in \overline{I\left(K_{0}\right)}\right\}=\inf \left\{r \in \mathbb{R}: \quad(r, a) \in I\left(K_{0}\right)\right\} \\
& =\min \{r \in \mathbb{R}: \quad(r, a) \in \operatorname{epi} \psi\} .
\end{aligned}
$$

Proof.

(a) Let $a \in \operatorname{ri}(\operatorname{dom} \bar{\psi})$ and take any $r \in \mathbb{R}$ satisfying $\bar{\psi}(a)<r<+\infty$. Then $(r, a) \in \operatorname{ri}(\operatorname{epi} \bar{\psi})$, and by (3.5), $\psi(a)<r$, implying $a \in \operatorname{dom} \psi$. This proves that $\operatorname{ri}(\operatorname{dom} \bar{\psi}) \subseteq$ dom $\psi$, showing the desired result. This also proves that $\bar{\psi}(a)=\psi(a)$ for all $a \in \operatorname{ri}(\operatorname{dom} \bar{\psi})$.

Let us check the second equality. Since $\operatorname{dom} \psi \subseteq \operatorname{dom} \bar{\psi}$, we obtain

$$
\overline{\operatorname{dom} \psi} \subseteq \overline{\operatorname{dom} \bar{\psi}}=\overline{\operatorname{ri}(\operatorname{dom} \bar{\psi})}=\overline{\operatorname{ri}(\operatorname{dom} \psi)}=\overline{\operatorname{dom} \psi},
$$

and the conclusion follows.

The last part is a consequence of $(a)$ and (3.6).

(b) For $a \in \operatorname{ri}(\operatorname{dom} \psi)$, one obtains

$$
\begin{aligned}
\psi(a) & =\bar{\psi}(a)=\min \{r \in \mathbb{R}:(r, a) \in \overline{\operatorname{epi} \psi}\}=\min \left\{r \in \mathbb{R}:(r, a) \in \overline{I\left(K_{0}\right)}\right\} \\
& \leq \inf \{r \in \mathbb{R}:(r, a) \in \operatorname{epi} \psi\} \leq \inf \left\{r \in \mathbb{R}:(r, a) \in I\left(K_{0}\right)\right\} \doteq r_{0} .
\end{aligned}
$$

Assume that $\psi(a)<r_{0}$. There exists $r_{k} \downarrow \psi(a)$ such that $\left(r_{k}, a\right) \in$ epi $\psi$. By $(a)$ of the previous proposition, we get $\left(r_{k}+\frac{1}{k}, a\right) \in I\left(K_{0}\right)$ for all $k \in \mathbb{N}$. This means that $r_{0} \leq r_{k}+\frac{1}{k}$, which implies $r_{0} \leq \psi(a)$, which is impossible, proving that $\psi(a)=r_{0}$, and the conclusion follows.

Remark 3.4. From (b) of Corollary 3.3, we obtain an existence result to problem $(P(a))$ (see (1.1)), namely, if $a \in \operatorname{ri}\left(g\left(C_{0}\right)+W\right), \psi(a)>-\infty$, and $I\left(K_{0}\right)$ is closed, then $(P(a))$ admits at least a solution.

Conditions implying the closedness of $I\left(K_{0}\right)$ are given in Theorem 2.1; whereas the nonemptiness of $\mathcal{H}$ yields $\psi(a)>-\infty$, as Theorem 7.1 shows.

Theorems 3.1 and 3.2 lead to the following characterization of lower semicontinuity of $\psi$ at 0 . Certainly, by Corollary $3.3, \psi$ is lsc in ri(dom $\psi$ ).

Proposition 3.5. Assume that $\psi(0)<+\infty$ and that the assumptions of Theorem 3.2 hold. Then,

(a) if $\psi(0)>-\infty$,

$$
\begin{aligned}
\bar{\psi}(0)= & \psi(0) \Longleftrightarrow\left[\overline{I\left(K_{0}\right)}-\psi(0)(1,0)\right] \cap\left(-\mathbb{R}_{++} \times\{0\}\right)=\emptyset \\
& \Longleftrightarrow\left[\operatorname{cone}\left(\overline{I\left(K_{0}\right)}-\psi(0)(1,0)\right)\right] \cap\left(-\mathbb{R}_{++} \times\{0\}\right)=\emptyset .
\end{aligned}
$$

(b) $\psi(0)=-\infty$ if and only if

$$
\left[I\left(K_{0}\right)-\rho(1,0)\right] \cap-\left(\mathbb{R}_{++} \times\{0\}\right) \neq \emptyset \quad \forall \rho \in \mathbb{R} .
$$

Proof.

(a) The proof follows by noticing that $\overline{I\left(K_{0}\right)}=$ epi $\bar{\psi}$.

(b) To prove this, simply consider the definition of $\psi(0)$. 
We now characterize the zero duality gap for our problem $(P(0))$ in terms of the lower semicontinuity of $\psi$ at 0 . In particular, if $0 \in \operatorname{ri}\left(g\left(C_{0}\right)+W\right)$, then there is no duality gap.

TheOREm 3.6. Assume that $W$ is additionally a cone, and $\psi(0)<+\infty$; then

(a) $v_{D}=\bar{\psi}(0)$;

(b) the duality gap between $(P(0))$ and (3.1) is zero, i.e., $\psi(0)=v_{D}$ if and only if $\psi(0)=\bar{\psi}(0)$.

Proof.

(a) In view of Theorem 3.1, we need to check that $\psi^{* *}(0)=\bar{\psi}(0)$. If $\bar{\psi}(0)=$ $-\infty$ then $\psi^{* *}(0)=-\infty$ since $\psi^{* *} \leq \bar{\psi}$. If $\bar{\psi}(0) \in \mathbb{R}$, due to the lower semicontinuity and convexity of $\bar{\psi}$, we conclude that $\bar{\psi}(=\overline{\mathrm{co}} \psi)$ never takes the value $-\infty$, and therefore $(\overline{\mathrm{co}} \psi=) \bar{\psi}=\psi^{* *}$.

(b) The proof follows from (a).

We will see next that the condition $0 \in \operatorname{ri}\left(g\left(C_{0}\right)+W\right)$ not only implies zero duality gap but also the existence of a solution for the dual problem provided $\psi(0)$ is finite. This is due to the important result derived from the convexity of $\psi$ (see Corollary 3.3): the nonemptiness of $\partial \psi(a)$ whenever $a \in \operatorname{ri}(\operatorname{dom} \psi)=\operatorname{ri}\left(g\left(C_{0}\right)+W\right)$.

Thus, on combining the previous theorem and Corollary 3.3, we establish the following result on strong duality for $(P(0))$ without any coercivity or convexity assumption.

Corollary 3.7. Assume that $\psi(0) \in \mathbb{R}$, with $W$ being additionally a cone, $K(0) \neq \emptyset$, and $0 \in \operatorname{ri}\left(g\left(C_{0}\right)+W\right)$. Then, there exists $\lambda_{0} \in W^{*}$ such that

$$
\inf _{z \in K(0)} \int_{0}^{1} f_{0}(t, z(t)) d t=\inf _{z \in \mathcal{Z}} \int_{0}^{1}\left[f_{0}(t, z(t))+\left\langle\lambda_{0}, g_{0}(t, z(t))\right\rangle\right] d t .
$$

For such $\lambda_{0}$, we have

$$
\inf _{z \in K(0)} \int_{0}^{1} f_{0}(t, z(t)) d t=\inf _{\substack{\left\langle\lambda_{0}, g(z)\right\rangle \leq 0 \\ z \in \mathcal{Z}}} \int_{0}^{1} f_{0}(t, z(t)) d t .
$$

Hence,

$$
\bar{z} \text { solves }(P(0)) \Longleftrightarrow\left\{\begin{array}{c}
\left\langle\lambda_{0}, \int_{0}^{1} g_{0}(t, \bar{z}(t)) d t\right\rangle=0, \\
\int_{0}^{1} f_{0}(t, \bar{z}(t)) d t=\inf _{z \in \mathcal{Z}} \int_{0}^{1}\left[f_{0}(t, z(t))+\left\langle\lambda_{0}, g_{0}(t, z(t))\right\rangle\right] d t .
\end{array}\right.
$$

Proof. By the previous theorem and Corollary 3.3, we get the zero duality gap. Moreover, since $\psi(0)$ is finite and $0 \in \operatorname{ri}(\operatorname{dom} \psi)=\operatorname{ri}\left(g\left(C_{0}\right)+W\right)$, a simple application of a convex separation theorem, allows us to conclude that $\partial \psi(0) \neq \emptyset$. Let $\lambda^{*} \in \partial \psi(0)$. Then, $\psi(a)-\psi(0) \geq\left\langle\lambda^{*}, a\right\rangle$ for all $a \in \mathbb{R}^{m}$. Since $W$ is a convex cone, we get $K(0) \subseteq K(a)$ for all $w \in W$; this along with the previous inequality implies that $\left\langle\lambda^{*}, a\right\rangle \leq 0$ for all $a \in W$, yielding $-\lambda^{*} \in W^{*}$. We need only to check that $-\lambda^{*}$ is a solution to problem (3.1).

Let us take any $z \in \mathcal{Z}$. For $a=\int_{0}^{1} g_{0}(t, z(t)) d t \in \mathbb{R}^{m}$, we obtain

$$
\int_{0}^{1} f_{0}(t, z(t)) d t-\left\langle\lambda^{*}, \int_{0}^{1} g_{0}(t, z(t)) d t\right\rangle \geq \psi(a)-\left\langle\lambda^{*}, a\right\rangle \geq \psi(0) \quad \forall z \in \mathcal{Z},
$$

which proves one inequality in (3.11) for $\lambda_{0}=-\lambda^{*}$; the other is trivial. 
Equality (3.12) and the remaining equivalence are not difficult to check.

In case $g_{0}(t, z)=z$ and $\mathcal{Z}=L^{1}\left([0,1], \mathbb{R}^{n}\right)$, we obtain $g\left(L^{1}\left([0,1], \mathbb{R}^{m}\right)\right)=\mathbb{R}^{m}$. Thus, $0 \in \operatorname{ri}\left(g\left(C_{0}\right)+W\right)$ trivially holds whatever $W$ is.

Related strong duality results were established in $[3,14]$.

Remark 3.8. Example 7.7 shows that the single condition $a \in \operatorname{ri}(\operatorname{dom} \psi)$ does not imply, in general, existence of solutions even when the optimal value is finite.

4. The subdifferential of the value function and necessary optimality conditions. Our aim in this section is to exploit the convexity property of the value function $\psi$ in order to compute its subdifferential; we know that $\partial \psi(a)$ is nonempty, convex, and compact whenever $a \in \operatorname{ri}(\operatorname{dom} \psi)$.

To that purpose, with the same assumptions on $f_{0}, g_{0}, W$, and $Z$, let us consider problem $(P(0))$, i.e., (1.1) with $a=0$, and its associated Hamiltonian function $H$ : $[0,1] \times \mathbb{R}^{m} \rightarrow \mathbb{R} \cup\{ \pm \infty\}$ defined by

$$
H(t, p) \doteq \sup _{\xi \in Z(t)}\left\{\left\langle p, g_{0}(t, \xi)\right\rangle-f_{0}(t, \xi)\right\}
$$

Obviously $H(t, \cdot)$ is convex and lsc for all $t \in[0,1]$, and because of (1.4), we have for all $p \in \mathbb{R}^{m}$

$$
H(t, p) \geq\left\langle p, g_{0}\left(t, z_{0}(t)\right)\right\rangle-f_{0}\left(t, z_{0}(t)\right) \text { a.e. } t \in[0,1] .
$$

Set

$$
\mathcal{U} \doteq\left\{(x, z) \in W_{m}^{1,1} \times L^{1}: \dot{x}(t)=g_{0}(t, z(t)), z(t) \in Z(t) \text {, a.e. } t \in[0,1], x(0)=0\right\} .
$$

The next theorem does not require that $W$ be a cone as in Corollary 3.7.

TheOREM 4.1. Let $\bar{z} \in K(0)$. Then the following assertions are equivalent:

(a) $p \in \partial \psi(0)$ and $\bar{z}$ solves $(P(0))$;

(b) $p \in N\left(W ;-\int_{0}^{1} g_{0}(t, \bar{z}(t)) d t\right)$ and

$$
H(t, p)=\left\langle p, g_{0}(t, \bar{z}(t))\right\rangle-f_{0}(t, \bar{z}(t)) \quad \text { a.e. } t \in[0,1] .
$$

Proof. (a) $\Rightarrow$ (b): Let $p \in \partial \psi(0)$ or, equivalently

$$
\langle p, a\rangle \leq \psi(a)-\psi(0) \quad \forall a \in \mathbb{R}^{m} .
$$

For any fixed $w \in W$, set $a \doteq \int_{0}^{1} g_{0}(t, \bar{z}(t)) d t+w$. Then, we have $\int_{0}^{1} g_{0}(t, \bar{z}(t)) d t \in$ $-W+a$. Replacing $a$ in (4.4) and taking into account that $\bar{z}$ is a solution to $(P(0))$, we get

$$
\left\langle p, w+\int_{0}^{1} g_{0}(t, \bar{z}(t)) d t\right\rangle \leq 0
$$

proving the first result in (b).

To establish (4.3), pick any $z \in L^{1}$, with $z(t) \in Z(t)$ a.e. $t \in[0,1]$. Then

$$
\int_{0}^{1} g_{0}(t, z(t)) d t \in-W+a
$$

where $a=\int_{0}^{1} g_{0}(t, z(t)) d t-\int_{0}^{1} g_{0}(t, \bar{z}(t)) d t$. Using (4.4), we obtain

$$
\left\langle p, \int_{0}^{1} g_{0}(t, z(t)) d t-\int_{0}^{1} g_{0}(t, \bar{z}(t)) d t\right\rangle \leq \int_{0}^{1} f_{0}(t, z(t)) d t-\psi(0) .
$$

Copyright $@$ ( ) by SIAM. Unauthorized reproduction of this article is prohibited. 
Thus, since $\bar{z}$ is a solution of the problem $(P(0))$ we have $\psi(0)=\int_{0}^{1} f_{0}(t, \bar{z}(t)) d t$ and hence $\bar{z}$ solves the problem

$$
\min \left\{\int_{0}^{1} f_{0}(t, z(t)) d t-\left\langle p, \int_{0}^{1} g_{0}(t, z(t)) d t\right\rangle: z \in \mathcal{Z}\right\}
$$

or, equivalently, $(\bar{x}, \bar{z})$, where $\bar{x}(t)=\int_{0}^{t} g_{0}(s, \bar{z}(s)) d s$, is a solution of the following problem

$$
\min _{(x, z) \in \mathcal{U}} \int_{0}^{1} f_{0}(t, z(t)) d t-\langle p, x(1)\rangle .
$$

The maximum principle (Theorem 6.1 in [10]) yields $q \in W_{m}^{1,1}$ such that

$$
\dot{q}(t)=0 \text { a.e. } t \in[0,1], \quad-q(1) \in \partial\langle-p, \cdot\rangle(\bar{x}(1))=\{-p\},
$$

and

$$
H(t, p)=\left\langle p, g_{0}(t, \bar{z}(t))\right\rangle-f_{0}(t, \bar{z}(t)) \text { a.e. } t \in[0,1],
$$

proving (4.3).

(b) $\Rightarrow$ (a): From (4.3), we obtain for all $z \in \mathcal{Z}$,

$$
\left\langle p, g_{0}(t, z(t))\right\rangle-f_{0}(t, z(t)) \leq\left\langle p, g_{0}(t, \bar{z}(t))\right\rangle-f_{0}(t, \bar{z}(t)) \text { a.e. } t \in[0,1]
$$

and, hence,

$$
\begin{aligned}
\left\langle p, \int_{0}^{1} g_{0}(t, z(t)) d t\right\rangle-\int_{0}^{1} f_{0}(t, & z(t)) d t \\
& \leq\left\langle p, \int_{0}^{1} g_{0}(t, \bar{z}(t)) d t\right\rangle-\int_{0}^{1} f_{0}(t, \bar{z}(t)) d t .
\end{aligned}
$$

Now let $z \in K(0)$. Then $\int_{0}^{1} g_{0}(t, z(t)) d t \in-W$, and by the first part in $(b)$,

$$
\left\langle p,-\int_{0}^{1} g_{0}(t, z(t)) d t+\int_{0}^{1} g_{0}(t, \bar{z}(t)) d t\right\rangle \leq 0 .
$$

This along with (4.6) implies that for all $z \in K(0)$,

$$
\int_{0}^{1} f_{0}(t, z(t)) d t \geq \int_{0}^{1} f_{0}(t, \bar{z}(t)) d t
$$

ensuring that $\bar{z}$ is a solution to $(P(0))$.

We now prove $p \in \partial \psi(0)$. Take any $a \in \mathbb{R}^{m}$ satisfying $K(a) \neq \emptyset$. Then for all $z \in K(a)$, we have $z \in \mathcal{Z}$ and $\int_{0}^{1} g_{0}(t, z(t)) d t-a \in-W$. On the one hand, the first part of (b) ensures that

$$
\left\langle p,-\int_{0}^{1} g_{0}(t, z(t)) d t+a+\int_{0}^{1} g_{0}(t, \bar{z}(t)) d t\right\rangle \leq 0 .
$$

It follows from (4.6)

$$
\int_{0}^{1} f_{0}(t, z(t)) d t \geq \int_{0}^{1} f_{0}(t, \bar{z}(t)) d t+\langle p, a\rangle
$$

Copyright (c) by SIAM. Unauthorized reproduction of this article is prohibited. 
and as $\psi(0)=\int_{0}^{1} f_{0}(t, \bar{z}(t)) d t$ and $z \in K(a)$ is arbitrary, we get

$$
\psi(a)-\psi(0) \geq\langle p, a\rangle
$$

or, equivalently, $p \in \partial \psi(0)$.

We recover Proposition 5.8 in [3].

Corollary 4.2. Assume that $0 \in \operatorname{ri}\left(g\left(C_{0}\right)+W\right)$ and $\bar{z} \in K(0)$. Then the following assertions are equivalent:

(a) $\bar{z}$ is a solution to $(P(0))$;

(b) there exists $p \in N\left(W ;-\int_{0}^{1} g_{0}(t, \bar{z}(t)) d t\right)$ such that

$$
H(t, p)=\left\langle p, g_{0}(t, \bar{z}(t))\right\rangle-f_{0}(t, \bar{z}(t)) \quad \text { a.e. } t \in[0,1] .
$$

The set of $p$ satisfying $(b)$ is $\partial \psi(0)$.

Proof. It suffices to see that our assumption $0 \in \operatorname{ri}(\operatorname{dom} \psi)$ ensures the existence of $p \in \partial \psi(0)$ and then we apply Theorem 4.1.

5. Local minima for the problem (1.1) are global. The aim of the section is to show that the previous assumptions (except assumption (1.5)) together with the following constraint qualification

$$
0 \in \operatorname{int}\left[g\left(C_{0}\right)+W\right]
$$

ensure that each local minimum for $(P(0))$ is in fact global. Here, we recall that

$$
C_{0}=\{z \in \mathcal{Z}: f(z)<+\infty\} .
$$

THEOREM 5.1. Let $f_{0}$ and $g_{0}$ satisfy the previous measurability, lower semicontinuity, and continuity assumptions and let $W$ be closed and convex. Then, under the qualification condition (5.1), each local minimum for $(P(0))$ is global.

Before giving the proof of this theorem, we establish a result concerning the necessary optimality conditions of $(P(0))$. These necessary conditions are expressed in terms of the limiting Fréchet (or Mordukhovich [12]) normal cone that we begin by recalling here.

Let $C$ be a closed subset of $\mathbb{R}^{n}$ containing some point $c$. The Fréchet normal cone to $C$ at $c$ is the set

$$
\hat{N}(C ; c) \doteq\left\{\xi \in \mathbb{R}^{n}: \liminf _{x \in C \rightarrow c} \frac{\langle-\xi, x-c\rangle}{\|x-c\|} \geq 0\right\} .
$$

The normal cone to $C$ at $c$ is the set

$$
N(C ; c) \doteq \limsup _{x \in C \rightarrow c} \hat{N}(C ; x) .
$$

LEMma 5.2. If $\bar{z}$ is a local solution for $(P(0))$, then there exist $p \in \mathbb{R}^{m}$ and $\lambda \in\{0,1\}$ with $(p, \lambda) \neq(0,0)$, such that $p \in N\left(W ;-\int_{0}^{1} g_{0}(t, \bar{z}(t)) d t\right)$ and

$$
\left\langle p, g_{0}(t, \bar{z}(t))\right\rangle-\lambda f_{0}(t, \bar{z}(t))=\max _{z \in Z(t) \cap \operatorname{dom} f(t, \cdot)}\left[\left\langle p, g_{0}(t, z)\right\rangle-\lambda f_{0}(t, z)\right] \text { a.e. } t \in[0,1] .
$$

Proof. We define the functions $\ell: \mathbb{R}^{m} \times \mathbb{R}^{n} \times \mathbb{R}^{m} \times \mathbb{R}^{n} \rightarrow \mathbb{R} \cup\{+\infty\}$ and $L:[0,1] \times \mathbb{R}^{m} \times \mathbb{R}^{n} \times \mathbb{R}^{m} \times \mathbb{R}^{n} \rightarrow \mathbb{R} \cup\{+\infty\}$ by

$$
\begin{aligned}
\ell\left(s_{1}, s_{2}, s_{3}, s_{4}\right) & =\iota_{\{0\}}\left(s_{1}\right)+\iota_{\{0\}}\left(s_{2}\right)+\iota_{-W}\left(s_{3}\right), \\
L(t, x, y, u, v) & = \begin{cases}f_{0}(t, v) & \text { if } v \in Z(t), u=g_{0}(t, v), \\
+\infty & \text { otherwise }\end{cases}
\end{aligned}
$$

Copyright $@$ by SIAM. Unauthorized reproduction of this article is prohibited. 
Let us note that, as $L$ does not depend on $(x, y)$, then for each element $(x, y, u, v, r) \in$ epi $L(t, \cdot)$ we have

$$
\left(\beta, x^{*}, y^{*}, u^{*}, v^{*}\right) \in N(\operatorname{epi} L(t, \cdot) ;(x, y, u, v, r)) \Rightarrow x^{*}=0, y^{*}=0 .
$$

Put $\bar{x}(t)=\int_{0}^{t} g(\tau, \bar{z}(\tau)) d \tau$ and $\bar{y}(t)=\int_{0}^{t} \bar{z}(\tau) d \tau$. Since $\bar{z}$ is a local solution of the problem $(P(0))$, then $(\bar{x}, \bar{y})$ is a local solution of the following Bolza problem

$$
\left\{\min _{(x, y) \in W_{m}^{1,1} \times W_{n}^{1,1}} \int_{0}^{1} L(t, x(t), y(t), \dot{x}(t), \dot{y}(t)) d t+\ell(x(0), y(0), x(1), y(1))\right.
$$

It is not difficult to show that all the assumptions of Theorem 4.1.1 in [4] are satisfied and this theorem asserts the existence of an $\operatorname{arc} q=\left(p, p_{0}\right) \in W_{m}^{1,1} \times W_{n}^{1,1}$ and $\lambda \in\{0,1\}$ such that

1. $(q, \lambda) \neq 0$

2. $-p(1) \in N(-W ; \bar{x}(1)), p_{0}(1)=0$;

3. $\dot{q}(t) \in \overline{c o}\{R:(-\lambda, R, q) \in N(\operatorname{epi} L(t, \cdot) ; f(t, \bar{z}(t), \bar{x}(t), \bar{y}(t), \dot{\bar{x}}(t), \dot{\bar{y}}(t)))\}$ a.e. $t \in[0,1]$ and hence, due to $(5.2), \dot{q}=0$;

4. for almost every $t \in[0,1]$ and $(u, v) \in \operatorname{dom} L(t, \bar{x}(t), \bar{y}(t), \cdot, \cdot)$,

$$
\langle q(t),(u, v)-(\dot{\bar{x}}(t), \dot{\bar{y}}(t))\rangle \leq \lambda[L(t, \bar{x}(t), \bar{y}(t),(u, v))-L(t, \bar{x}(t), \bar{y}(t), \dot{\bar{x}}(t), \dot{\bar{y}}(t))] .
$$

Assertions 2 and 3 assert that $p_{0}=0$ and $p(t)=p(1$ ) for all $t \in[0,1]$ (we will put $p(t)=p$ for all $t \in[0,1])$, while assertion 4 implies the following:

$$
\left\langle p, g_{0}(t, \bar{z}(t))\right\rangle-\lambda f_{0}(t, \bar{z}(t))=\max _{z \in Z(t) \cap \operatorname{dom} f(t, \cdot)}\left[\left\langle p, g_{0}(t, z)\right\rangle-\lambda f_{0}(t, z)\right] \text { a.e. } t \in[0,1],
$$

and the proof of the lemma is finished because $-p \in N(-W ; \bar{x}(1))$ if and only if $p \in N(W ;-\bar{x}(1))$.

Now, we proceed to prove our theorem.

Proof. We will show that the multiplier $\lambda$ in Lemma 5.2 is equal to 1. Indeed, suppose that $\lambda=0$. Then, in particular

$$
\left\langle p, g_{0}(t, \bar{z}(t))\right\rangle \geq\left\langle p, g_{0}(t, z(t))\right\rangle \quad \forall z \in C_{0}
$$

and, hence, by integrating we get

$$
\langle p, g(z)-g(\bar{z})\rangle \leq 0 \quad \forall z \in C_{0} .
$$

On the other hand, $p \in N\left(W ;-\int_{0}^{1} g(t, \bar{z}(t)) d t\right)=N(W ;-g(\bar{z}))$ or, equivalently,

$$
\langle p, w+g(\bar{z})\rangle \leq 0 \quad \forall w \in W .
$$

Now adding the two last inequalities, we obtain that

$$
\langle p, g(z)+w\rangle \leq 0 \forall z \in C_{0} \forall w \in W .
$$

Using our constraint qualification (5.1), we conclude that $p=0$ and this contradicts $(p, \lambda) \neq(0,0)$. So $\lambda=1$ and hence the last equality in Lemma 5.2 can be written as

$$
\left\langle p, g_{0}(t, \bar{z}(t))\right\rangle-f_{0}(t, \bar{z}(t))=\max _{z \in Z(t)}\left[\left\langle p, g_{0}(t, z)\right\rangle-f_{0}(t, z)\right] \text { a.e. } t \in[0,1] .
$$

Copyright $@$ by SIAM. Unauthorized reproduction of this article is prohibited. 
We now apply Corollary 4.2 to conclude that $\bar{z}$ is a (global) solution to $(P(0))$, and the proof is completed.

Now, we can ask when the constraint qualification (5.1) is satisfied. In fact, it is easy to see that the following implication holds true for some $\rho>0$

$$
K(a) \neq \emptyset \quad \forall a \in B(0, \rho) \Rightarrow 0 \in \operatorname{int}\left[g\left(C_{0}\right)+W\right]
$$

provided $\mathcal{Z} \subseteq \operatorname{dom} f$.

Several characterizations concerning the nonemptiness of $K(a)$ around 0 will be presented in the next section.

6. The behavior of the set-valued mapping $\boldsymbol{K}$. The main intention of this section is to give sufficient conditions ensuring the nonemptiness and the behavior of the set-valued mapping $K$ considered in the previous section.

We will consider the set-valued mapping $\tilde{K}: \mathbb{R}^{m} \rightrightarrows W^{1,1} \times L^{1}$ defined by

$$
\begin{array}{r}
\tilde{K}(a) \doteq\left\{(x, u) \in W^{1,1} \times L^{1}: \dot{x}(t)=g_{0}(t, u(t)), u(t) \in Z(t), \text { a.e. } t \in[0,1],\right. \\
x(0)=0, x(1) \in-W+a\} .
\end{array}
$$

Theorem 6.1. Let $\bar{z} \in K(\bar{a})$ and put $\bar{x}(t)=\int_{0}^{t} g_{0}(\tau, \bar{z}(\tau)) d \tau$ for all $t \in[0,1]$.

$\mathcal{U} \doteq\left\{(x, u) \in W^{1,1} \times L^{1}: \dot{x}(t)=g_{0}(t, u(t)), u(t) \in Z(t)\right.$, a.e. $\left.t \in[0,1], x(0)=0\right\}$.

Suppose that $g_{0}(t, \cdot)$ is continuous for almost every $t \in[0,1]$ and $W$ is a closed set in $\mathbb{R}^{m}$. Let us consider the following assertions:

(i) There is no $p \in \mathbb{R}^{m}$, with $p \neq 0$, satisfying

$$
-p \in N(-W ; \bar{x}(1)-\bar{a}), \quad\left\langle p, g_{0}(t, \bar{z}(t))\right\rangle=\max _{z \in Z(t)}\left\langle p, g_{0}(t, z)\right\rangle \text { a.e. } t \in[0,1] .
$$

(ii) There exist $\alpha>0$ and $r>0$ such that

$$
d((x, u), \tilde{K}(a)) \leq \alpha d(x(1),-W+a)
$$

for all $(x, u) \in B((\bar{x}, \bar{z}), r) \cap \mathcal{U}$ and $a \in B(\bar{a}, r)$.

(iii) There exist $\alpha>0$ and $r>0$ such that

$$
\tilde{K}(a) \cap B((\bar{x}, \bar{z}), r) \subseteq \tilde{K}\left(a^{\prime}\right)+\alpha\left\|a-a^{\prime}\right\| B(0,1)
$$

for all $a, a^{\prime} \in B(\bar{a}, r)$.

(iv) There exists $r>0$ such that

$$
\forall a \in B(\bar{a}, r), \quad K(a) \neq \emptyset .
$$

Then (i) $\Rightarrow$ (ii) $\Rightarrow$ (iii) $\Rightarrow$ (iv). If moreover $W$ is convex, then (iv) $\Rightarrow$ (i).

Proof. The implications (ii) $\Rightarrow$ (iii) $\Rightarrow$ (iv) $\Rightarrow$ (i) are obvious. We establish only the implication (i) $\Rightarrow$ (ii): Suppose that (ii) does not hold. Then, there are sequences $\left(y_{k}, v_{k}\right) \in \mathcal{U}$, with $y_{k} \rightarrow \bar{x}$ and $v_{k} \rightarrow \bar{z}$, and $a_{k} \rightarrow \bar{a}$ such that for $k$ large enough

$$
d\left(\left(y_{k}, v_{k}\right), \tilde{K}\left(a_{k}\right)\right)>k d\left(y_{k}(1),-W+a_{k}\right) .
$$

Copyright (C) by SIAM. Unauthorized reproduction of this article is prohibited. 
It follows that $\left(y_{k}, v_{k}\right) \notin \tilde{K}\left(a_{k}\right)$. Let us consider the function $f_{k}: W^{1,1} \times L^{1} \rightarrow \mathbb{R}$ defined by

$$
f_{k}(x, u)=d\left(x(1),-W+a_{k}\right) .
$$

Put $\varepsilon_{k}=\sqrt{f_{k}\left(y_{k}, v_{k}\right)}>0$ and $\lambda_{k}=\min \left\{k \varepsilon_{k}^{2}, \varepsilon_{k}\right\}$. Then $\varepsilon_{k} \rightarrow 0$ and $\lambda_{k} \rightarrow 0$. Obviously,

$$
f_{k}\left(y_{k}, v_{k}\right) \leq \inf _{(y, u) \in \mathcal{U}} f_{k}(y, u)+\varepsilon_{k}^{2}
$$

Our assumption on $g_{0}$ ensures that $\mathcal{U}$ is closed in $W^{1,1} \times L^{1}$. Now, applying the Ekeland variational principle one gets the existence of $\left(x_{k}, u_{k}\right) \in \mathcal{U}$ such that

$$
\begin{aligned}
\left\|\left(x_{k}, u_{k}\right)-\left(y_{k}, v_{k}\right)\right\| & \leq \lambda_{k}, \\
f_{k}\left(x_{k}, u_{k}\right) & \leq f_{k}(x, u)+s_{k}\left\|(x, u)-\left(x_{k}, u_{k}\right)\right\| \quad \forall(x, u) \in \mathcal{U},
\end{aligned}
$$

where $s_{k}=\frac{\varepsilon_{k}^{2}}{\lambda_{k}}$. Using the inequality $(6.3)$, we obtain that $\left(x_{k}, u_{k}\right)$ is a solution to the following optimal control problem of Mayer type:

$$
\left\{\begin{array}{c}
\min _{(x, u) \in \mathcal{U}} d\left(x(1)-a_{k},-W\right)+s_{k}\left\|x(0)-x_{k}(0)\right\| \\
+s_{k} \int_{0}^{1}\left[\left\|g_{0}(t, u(t))-g_{0}\left(t, u_{k}(t)\right)\right\|+\left\|u(t)-u_{k}(t)\right\|\right] d t .
\end{array}\right.
$$

The maximum principle yields an arc $p_{k} \in W^{1,1}\left([0,1], \mathbb{R}^{n}\right)$ such that

$$
\dot{p}_{k}(t)=0 \text { a.e. } t \in[0,1], \quad-p_{k}(1) \in \partial d\left(\cdot-a_{k},-W\right)\left(x_{k}(1)\right),
$$

and for a.e. $t \in[0,1]$

$$
\begin{aligned}
& \left\langle p_{k}(t), g_{0}\left(t, u_{k}(t)\right)\right\rangle \\
& \quad=\max _{u \in Z(t)}\left\langle p_{k}(t), g_{0}(t, u)\right\rangle-s_{k}\left[\left\|u-u_{k}(t)\right\|+\left\|g_{0}(t, u)-g_{0}\left(t, u_{k}(t)\right)\right\|\right]
\end{aligned}
$$

where $\partial d\left(\cdot-a_{k},-W\right)\left(x_{k}(1)\right)$ is the limiting subdifferential [12] of the distance function to the set $-W+a_{k}$. From (6.1) and (6.2) it follows that $x_{k}(1) \notin-W+a_{k}$, and this implies that $\left\|p_{k}(1)\right\|=1$ (see [11]). Since $\lambda_{k} \rightarrow 0,(6.2)$ together with $v_{k} \rightarrow \bar{z}$ ensure that $u_{k} \rightarrow \bar{z}$ in $L^{1}\left([0,1], \mathbb{R}^{n}\right)$ and hence there exists a subsequence $\left(u_{\varphi(k)}\right)$ of $\left(u_{k}\right)$ such that

$$
u_{\varphi(k)}(t) \rightarrow \bar{z}(t) \text { a.e. } t \in[0,1]
$$

and extracting a subsequence, if necessary, we may also assume that $p_{\varphi(k)}(1) \rightarrow p$ with $p \neq 0$. Because of the closedness of the limiting subdifferential [12], $-p \in$ $\partial d(\cdot,-W)\left(\int_{0}^{1} g_{0}(t, \bar{z}(t)) d t-\bar{a}\right) \subseteq N\left(-W ; \int_{0}^{1} g_{0}(t, \bar{z}(t)) d t-\bar{a}\right)$. Now, having in mind that $s_{\varphi(k)}=\max \left(\frac{1}{\varphi(k)}, \varepsilon_{\varphi(k)}\right)$, we get $s_{\varphi(k)} \rightarrow 0$. On the other hand, relation (6.4) and the continuity of $g_{0}(t, \cdot)$ ensure that

$$
\left\langle p, g_{0}(t, \bar{z}(t))\right\rangle=\max _{u \in Z(t)}\left\langle p, g_{0}(t, u)\right\rangle \text { a.e. } t \in[0,1]
$$

and this contradicts (i). 
7. Computing the value function via the Hamiltonian and existence of solutions. This section is devoted to provide conditions under which the value function $\psi$ is minorized by an affine linear function. As a consequence, we find a formula for $\psi^{*}$ and so of $\psi$. To that end, let us define the function $G: \mathbb{R}^{m} \rightarrow \mathbb{R} \cup\{ \pm \infty\}$ by

$$
G(p)=\int_{0}^{1} H(t, p) d t
$$

where, $H$ is the Hamiltonian function defined as in (4.1). It follows that $G$ is lsc and convex. Using Theorem 2.2 in [8], we may rewrite the function $G$ as follows:

$$
G(p)=\sup _{z \in \mathcal{Z}} \int_{0}^{1}\left[\left\langle p, g_{0}(t, z(t))\right\rangle-f_{0}(t, z(t))\right] d t .
$$

The next function that will be useful in the sequel is

$$
G_{0}(p)=\left\{\begin{array}{lll}
G(p) & \text { if } & p \in-W^{*} \\
+\infty & \text { if } & p \notin-W^{*}
\end{array}\right.
$$

Thus, $G_{0}$ is lsc and convex. Set

$$
\mathcal{H} \doteq\left\{p \in-W^{*}: H(\cdot, p) \in L^{1}([0,1], \mathbb{R})\right\} .
$$

Then, by (4.2), $\mathcal{H}$ is convex and

$$
p \in \mathcal{H} \Longleftrightarrow p \in-W^{*} \text { and } p \in \operatorname{dom} G \Longleftrightarrow p \in \operatorname{dom} G_{0} .
$$

The next result generalizes and extends Theorem 3.2 in [6], where the case $W=\{0\}$ and $g_{0}(t, z)=z$ is considered.

TheOrem 7.1. Assume that $W$ is additionally a cone and $\mathcal{H} \neq \emptyset$. The following statements hold.

(a) $\psi(a) \geq\left\langle p^{*}, a\right\rangle-G\left(p^{*}\right)>-\infty \forall a \in \mathbb{R}^{m} \forall p^{*} \in \mathcal{H}$; consequently, $\bar{\psi}=\overline{\mathrm{co}} \psi=$ $\psi^{* *}$.

(b) Assume that (1.4) and (1.5) are satisfied. Then $\psi^{*}(p)=G_{0}(p) \forall p \in \mathbb{R}^{m}$, and so $\psi^{* *}=\bar{\psi}=G_{0}^{*}$, which gives $v_{D}=G_{0}^{*}(0)$.

(c) $\psi(a)=G_{0}^{*}(a)$ for all $a \in \operatorname{ri}\left(g\left(C_{0}\right)+W\right)$.

Proof.

(a) Let $p^{*} \in \mathcal{H}$. We have

$$
G\left(p^{*}\right) \doteq \int_{0}^{1} H\left(t, p^{*}\right) d t \geq \int_{0}^{1}\left\langle p^{*}, g_{0}(t, z(t))\right\rangle d t-\int_{0}^{1} f_{0}(t, z(t)) d t \quad \forall z \in \mathcal{Z} .
$$

Take any $a \in \mathbb{R}^{m}$ such that $K(a) \neq \emptyset$. Then for $z \in K(a)$ there exists $p \in W$ such that

$$
\int_{0}^{1} f_{0}(t, z(t)) d t \geq \int_{0}^{1}\left\langle p^{*}, g_{0}(t, z(t))\right\rangle d t-\int_{0}^{1} H\left(t, p^{*}\right) d t \geq \int_{0}^{1}\left\langle p^{*}, a-p\right\rangle d t-G\left(p^{*}\right) .
$$

Since $p^{*} \in-W^{*}$ we have

$$
\psi(a) \geq \int_{0}^{1}\left\langle p^{*}, a\right\rangle d t-G\left(p^{*}\right)=\left\langle p^{*}, a\right\rangle-G\left(p^{*}\right) .
$$

Copyright $@$ by SIAM. Unauthorized reproduction of this article is prohibited. 
(b) Let $\lambda \in \mathbb{R}^{m}$. Then,

$$
\begin{aligned}
\psi^{*}(\lambda) & =\sup _{a \in \mathbb{R}^{n}}\{\langle\lambda, a\rangle-\psi(a)\}=\sup _{\{a: K(a) \neq \emptyset\}} \sup _{z \in K(a)}\{\langle\lambda, a\rangle-f(z)\} \\
& =\sup _{z \in C_{0}, p \in W}\{\langle\lambda, g(z)\rangle+\langle\lambda, p\rangle-f(z)\} \\
& =\sup _{z \in C_{0}}\{\langle\lambda, g(z)\rangle-f(z)\}+\sup _{p \in W}\langle\lambda, p\rangle \\
& =\left\{\begin{array}{cc}
\sup _{z \in C_{0}}\{\langle\lambda, g(z)\rangle-f(z)\} & \text { if } \lambda \in-W^{*}, \\
+\infty & \text { if } \lambda \notin-W^{*}
\end{array}\right.
\end{aligned}
$$

By definition

$$
H(t, \lambda) \geq\left\langle\lambda, g_{0}(t, z(t))\right\rangle-f_{0}(t, z(t)) \text { for a.e. } t \in[0,1] \forall z \in \mathcal{Z},
$$

so that for all $\lambda \in \mathbb{R}^{m}$,

$$
G(\lambda) \geq \int_{0}^{1}\left[\left\langle\lambda, g_{0}(t, z(t))\right\rangle-f_{0}(t, z(t))\right] d t=\langle\lambda, g(z)\rangle-f(z) \forall z \in \mathcal{Z} .
$$

Hence, $G(\lambda) \geq \psi^{*}(\lambda)$ for all $\lambda \in-W^{*}$ because of (7.4). Suppose that $G(\lambda)>$ $\psi^{*}(\lambda)$ for some $\lambda \in-W^{*}$. Then there exists $z \in \mathcal{Z}$ such that

$$
\int_{0}^{1}\left\langle\lambda, g_{0}(t, z(t))\right\rangle d t-\int_{0}^{1} f_{0}(t, z(t)) d t>\psi^{*}(\lambda) .
$$

But relation (1.5) together with the last inequality ensure that $f(z)<\infty$ and, hence, $z \in C_{0}$ and this contradiction completes the proof of the equality $G(\lambda)=\psi^{*}(\lambda)$.

(c) It is a consequence of (b).

Next corollary, which is important by itself, provides another formula for the optimal value $\psi(0)$.

Corollary 7.2. Assume that $W$ is additionally a cone, $\mathcal{H} \neq \emptyset, 0 \in$ $\operatorname{ri}\left(g\left(C_{0}\right)+W\right),(1.4)$ and (1.5) are satisfied. Then there exists $p^{*} \in-W^{*}$ such that $\psi(0)=-G\left(p^{*}\right)$.

Proof. By assumption, it is known that there exists $p^{*} \in \partial \psi(0)$ and $\psi(0)=\bar{\psi}(0)$ by Corollary 3.3. Recall that by Theorem 3.2, the function $\psi$ is convex and by $(a)$ of Theorem 7.1 if follows that it is proper. Applying Theorem 23.5 in [16], we get

$$
p^{*} \in \partial \psi(0) \Leftrightarrow \psi(0)+\psi^{*}\left(p^{*}\right)=0 .
$$

Then

$$
\psi(0)=-\psi^{*}\left(p^{*}\right) .
$$

From Theorem 7.1 we also have that $\psi^{*}(p)=G_{0}(p)$ for all $p \in \mathbb{R}^{m}$ and $\psi(0)=G_{0}^{*}(0)$. Then, taking into account (7.5), we get

$$
\psi(0)=G_{0}^{*}(0)=-G_{0}\left(p^{*}\right) .
$$

Moreover, from (7.5) it also follows that $p^{*} \in \operatorname{dom} \psi^{*}=\operatorname{dom} G_{0}=\mathcal{H}$; therefore, $p^{*} \in-W^{*}$ and $G_{0}\left(p^{*}\right)=G\left(p^{*}\right)$. 
The following existence theorem subsumes Corollary 3.1 in [6].

TheOrem 7.3. Assume that $W$ is in addition a cone, $\mathcal{H} \neq \emptyset$, and (1.4) along with (1.5) hold. If $K_{0}$ is closed and the set epi $G_{0}^{*}$ contains no lines or extremal half-lines, then for every $a \in \mathbb{R}^{m}$ either $\psi(a)=+\infty$ or $(P(a))$ admits a solution, and the duality gap between $(P(0))$ and (3.1) is zero, i.e., $v_{D}=\psi(0)$.

Proof. By Theorem 2.1, $I\left(K_{0}\right)$ is closed since $\overline{I\left(K_{0}\right)}=$ epi $G_{0}^{*}$. Thus, $I\left(K_{0}\right)=$ $F\left(C_{0}\right)+\left(\mathbb{R}_{+} \times W\right)$ is closed and convex. From (3.4) we obtain

$$
\text { epi } \psi=F\left(C_{0}\right)+\left(\mathbb{R}_{+} \times W\right),
$$

which implies that $(P(a))$ admits a solution for every $a \in \mathbb{R}^{m}$ satisfying $K(a) \neq \emptyset$, and $\psi=\bar{\psi}$. Hence $v_{D}=\psi^{* *}(0)=\bar{\psi}(0)$ by Theorem 3.6(a).

Unfortunately we were unable to check that $K_{0}$ is closed in this general setting. However if $W=\{0\}$ and $g_{0}(t, z)=z, K_{0}$ is closed as one can check it directly. This case was considered in [6].

Next result follows a reasoning similar to that applied in Proposition 3.1 in [6].

THEOREM 7.4. Assume that $\mathcal{H}$ is a nonempty open set, $g\left(C_{0}\right)+W$ is open, where $W$ is additionally a cone, $K_{0}$ is closed, and (1.4) and (1.5) are satisfied. Then for every $a \in \mathbb{R}^{m}$ either $\psi(a)=+\infty$ or $(P(a))$ admits a solution for every $a \in g\left(C_{0}\right)+W$.

Proof. We will show that the assumptions of Theorem 7.3 are fulfilled. To this aim, it is enough to show that epi $G_{0}^{*}$ contains no lines or extremal half-lines. Recall that by Theorem 3.4, $\overline{I\left(K_{0}\right)}=$ epi $\bar{\psi}$, and by Theorem 7.1(b), epi $G_{0}^{*}=\overline{I\left(K_{0}\right)}$. We first note that $\bar{\psi}(a) \neq-\infty$ for every $a \in \operatorname{dom} \bar{\psi}$, otherwise $\bar{\psi}(\bar{a})=-\infty$ for a given $\bar{a} \in \operatorname{dom} \bar{\psi}$ would imply $\bar{\psi}(a)=-\infty$ for every $a \in \operatorname{dom} \bar{\psi} \supseteq \operatorname{dom} \psi$, and this contradicts Theorem 7.1(a), recalling that $\psi(a)=\bar{\psi}(a)$ for every $a \in \operatorname{ri}(\operatorname{dom} \psi)$. This implies that $\overline{I\left(K_{0}\right)}$ cannot have any vertical line, i.e., a line of the form $(t, \bar{\gamma}), t \in \mathbb{R}$, where $\bar{\gamma} \in \mathbb{R}^{m}$.

We next show that $\overline{I\left(K_{0}\right)}$ cannot have any extremal vertical half-line, i.e., half-line of the form $(t, \bar{\gamma}), t \geq \bar{t} \in \mathbb{R}$.

Let us consider any point $(\bar{\psi}(\bar{a}), \bar{a}) \in \overline{I\left(K_{0}\right)}$ and let $H$ be any supporting hyperplane to $\overline{I\left(K_{0}\right)}$ at $(\bar{\psi}(\bar{a}), \bar{a})$. Let $D \doteq \overline{I\left(K_{0}\right)} \cap H$. We observe that $D$ may contain a vertical half-line but this cannot be extremal, since otherwise $(\bar{\psi}(\bar{a}), \bar{a})$ would be an extremal point of $\overline{I\left(K_{0}\right)}$, and by Theorem 3 in [13], it follows that $(\bar{\psi}(\bar{a}), \bar{a}) \in I\left(K_{0}\right) \subseteq$ epi $\psi$ : this is a contradiction since, being that dom $\psi=g\left(C_{0}\right)+W$ is open, then $\partial \psi(a)$ is compact for every $a \in \operatorname{dom} \psi$ so that epi $\psi$ cannot have any vertical supporting hyperplane. This shows that epi $G_{0}^{*}$ cannot have any vertical lines, nor vertical extremal half-lines. Next, we prove that it cannot have any nonvertical half-lines. Suppose that there exists a half-line $s \doteq\left\{\left(\alpha, \xi_{0}\right)+\eta(\beta, z), \eta \geq 0\right\}$ contained in bd(epi $\left.G_{0}^{*}\right), \xi_{0}, z \in \mathbb{R}^{m}, z \neq 0, \alpha, \beta \in \mathbb{R}$, and let $\Gamma(\xi) \doteq\langle q, \xi\rangle-\gamma_{0}, q \in \mathbb{R}^{m}, \gamma_{0} \in \mathbb{R}$ be a supporting hyperplane for epi $G_{0}^{*}$ containing the half-line $s$. Then $G_{0}^{*}(\xi) \geq \Gamma(\xi)$ for all $\xi \in \mathbb{R}^{m}$, which implies $G_{0}^{* *}(q)=G_{0}(q) \leq \Gamma^{*}(q)=\gamma_{0}$, yielding $q \in \mathcal{H}$. Moreover,

$$
G_{0}^{*}\left(\xi_{0}+\eta z\right)=\Gamma\left(\xi_{0}+\eta z\right) \quad \forall \eta \geq 0
$$

because $s$ is contained in the graph of $\Gamma$.

Let $p \in \mathcal{H}$; by the previous relations and recalling that $G_{0}$ is an lsc convex function, we get

$$
\begin{aligned}
G_{0}^{* *}(p) & =G_{0}(p)=\sup _{\xi \in \mathbb{R}^{m}}\left[\langle p, \xi\rangle-G_{0}^{*}(\xi)\right] \\
& \geq\left\langle p, \xi_{0}+\eta z\right\rangle-G_{0}^{*}\left(\xi_{0}+\eta z\right)=\left\langle p-q, \xi_{0}+\eta z\right\rangle+\gamma_{0} \quad \forall \eta \geq 0 .
\end{aligned}
$$

Copyright $@$ by SIAM. Unauthorized reproduction of this article is prohibited. 
Since $G_{0}(p)<\infty$, it follows that

$$
\langle p-q, z\rangle \leq 0 \quad \forall p \in \mathcal{H},
$$

which is impossible because $q \in \mathcal{H}=\operatorname{int} \mathcal{H}$ and $z \neq 0$. Thus bd(epi $G_{0}^{*}$ ) does not contain any half-line, and since epi $G_{0}^{*}$ is a proper closed convex set, this implies that it cannot contain any line too, which completes the proof.

Remark 7.5. By the previous proof it is possible to show that the assumption on the opennes of $\mathcal{H}$ can be replaced by the following: "for every $p \in \mathcal{H}$, the subdifferential of $G_{0}$ is either bounded or empty." Indeed, (7.6) yields

$$
G_{0}(p)-G_{0}(q) \geq G_{0}(p)-\gamma_{0} \geq\left\langle p-q, \xi_{0}+\eta z\right\rangle \quad \forall p \in \mathcal{H} \forall \eta \geq 0,
$$

i.e., $\xi_{0}+\eta z \in \partial G_{0}(q)$ for all $\eta \geq 0$. By the previous assumption we get a contradiction that completes the proof.

A particular situation where $g\left(C_{0}\right)+W$ is open occurs when $g\left(C_{0}\right)+W=\mathbb{R}^{m}$. It is satisfied if $g_{0}(t, z)=z$ and $C_{0}=L^{1}$, in which case $m=n$. Observe also that in such a situation $K_{0}$ is closed.

Next, three examples illustrate the validity of Theorem 7.3 showing that some of the assumptions are essential; whereas the fourth one shows an instance where Theorem 7.4 is applicable with $g\left(C_{0}\right)+W$ being an open set contained strictly in $\mathbb{R}^{m}$.

Example 7.6. Let us consider the problem

$$
\psi(a) \doteq \inf \left\{\int_{0}^{1}-[z(t)]^{2} d t \quad \text { s.t. } \quad \int_{0}^{1} z(t) d t=a, \quad z \in \mathcal{Z}\right\}
$$

where $Z(t) \doteq\left[\frac{1}{\sqrt{t}},+\infty\left[\right.\right.$ for $t \in(0,1]$. Here $W=\{0\}$. Since $z(t) \geq \frac{1}{\sqrt{t}}$ for a.e. $t \in[0,1]$, then

$$
a=\int_{0}^{1} z(t) d t \geq \int_{0}^{1} \frac{1}{\sqrt{t}} d t=2 \quad \forall z \in \mathcal{Z} .
$$

Obviously $a=2 \in \operatorname{bd}(\operatorname{dom} \psi)$ and

$$
\psi(2)=\int_{0}^{1}-\frac{1}{t} d t=-\infty .
$$

We actually have $\psi(a)=-\infty$ for all $a \geq 2$. Indeed, the function $z(t) \doteq \frac{a}{2 \sqrt{t}} \in K(a)$, for all $a \geq 2$, and

$$
\psi(a) \leq \int_{0}^{1}-\frac{a^{2}}{4 t} d t=-\infty
$$

Note that

$$
\bar{\psi}(a)=\psi(a)=\left\{\begin{array}{lll}
+\infty & \text { if } & a<2 \\
-\infty & \text { if } & a \geq 2
\end{array}\right.
$$

is convex; whereas $\psi^{* *} \equiv-\infty, \mathcal{H}=\emptyset$, and $G \equiv+\infty$.

Example 7.7. Consider the problem

$$
\psi(a) \doteq \inf \left\{\int_{0}^{1} e^{-\left[z_{1}(t) z_{2}(t)\right]^{2}} d t \quad \text { s.t. } \int_{0}^{1}\left[z_{2}(t)-z_{1}(t)\right]^{2} d t \leq a, \quad z \in \mathcal{Z}\right\}
$$

Copyright (c) by SIAM. Unauthorized reproduction of this article is prohibited. 
where $Z(t) \doteq\left(\mathbb{R}_{+} \times \mathbb{R}_{+}\right)$for $t \in[0,1]$. Here $W=\mathbb{R}_{+}$. Obviously $K(a)=\emptyset$ for $a<0$. Setting $\bar{z}_{1}(t)=\bar{z}_{2}(t)=c$ for all $t \in[0,1]$, then for every $a \geq 0$, and for every $c \geq 0$, $\bar{z} \in K(a)$. Obviously $a=0 \in \operatorname{bd}(\operatorname{dom} \psi)$ and

$$
0 \leq \psi(a) \leq \int_{0}^{1} e^{-c^{4}} d t=e^{-c^{4}} \quad \forall c \geq 0,
$$

which implies

$$
\psi(a)=0 \quad \forall a \geq 0 .
$$

Note that the infimum is never attained and

$$
\psi(a)=\left\{\begin{array}{rll}
+\infty & \text { if } & a<0, \\
0 & \text { if } & a \geq 0
\end{array}\right.
$$

is convex with $\psi=\bar{\psi}$. Here $\mathcal{H}=]-\infty, 0]$,

$$
G(p)=G_{0}(p)=\left\{\begin{array}{rll}
+\infty & \text { if } & p>0 \\
0 & \text { if } & p \leq 0
\end{array}\right.
$$

Thus $\psi=G_{0}^{*}$ as expected by Theorem 7.1. Moreover the assumptions of Theorems 2.1 (with $K_{0}$ instead of $K$ ) or 7.3 are not fulfilled since an extremal half-line belongs to $\overline{I\left(K_{0}\right)}=$ epi $\bar{\psi}$.

Example 7.8. Consider the problem

$$
\inf \left\{\int_{0}^{1} e^{-[z(t)]} d t \quad \text { s.t. } \quad \int_{0}^{1} \frac{z(t)}{1+z(t)} d t \leq a, \quad z \in \mathcal{Z}\right\},
$$

where $Z(t) \doteq\left[0,+\infty\left[\right.\right.$ for $t \in[0,1]$. Here $W=\mathbb{R}_{+}$. Obviously $K(a)=\emptyset$ for $a<0$.

Let $a \geq 1$. Setting $\bar{z}(t)=c \geq 0 \forall t \in[0,1]$, then for every $a \geq 1$, and for every $c \geq 0, \bar{z} \in K(a)$.

Then,

$$
0 \leq \psi(a) \leq \int_{0}^{1} e^{-c} d t=e^{-c} \quad \forall c \geq 0,
$$

which implies $\psi(a)=0$ for all $a \geq 1$. Note that the infimum is never attained for $a \geq 1$.

Let $0 \leq a<1$. In such a case we have $\psi(a)>0$. Indeed $\psi(a)=0$ if and only if there exists a sequence $z^{k} \in K(a)$ such that

$$
\lim _{k \rightarrow+\infty} z^{k}(t)=+\infty \text { for a.e. } t \in[0,1],
$$

but this would imply

$$
\lim _{k \rightarrow+\infty} \int_{0}^{1} \frac{z^{k}(t)}{1+z^{k}(t)} d t=1
$$

which contradicts that $z^{k} \in K(a)$ with $a<1$, for $k$ sufficiently large. Therefore,

$$
\psi(a)=\left\{\begin{array}{cll}
+\infty & \text { if } & a<0 \\
\psi(a)>0 & \text { if } & 0 \leq a<1 \\
0 \quad \text { if } & a \geq 1
\end{array}\right.
$$

Copyright (c) by SIAM. Unauthorized reproduction of this article is prohibited. 
Since $\operatorname{ri}(\operatorname{dom} \psi)=] 0,+\infty[$, then $\psi(a)=\bar{\psi}(a)$ for every $a \in] 0,+\infty[$. Observe that $(\psi(a), a)=(\psi(1), 1)$ is an extreme point of epi $\bar{\psi}$ with $a=1$ belonging to ri(dom $\psi)$ and such that the infimum value of $(P(1))$ is not attained.

We note that the assumptions of Theorem 2.1, or Theorem 7.3, are not fulfilled since an extremal half-line belongs to $\overline{I\left(K_{0}\right)}=$ epi $\bar{\psi}$.

The next instance exhibits a situation where dom $\psi$ is open without being the whole space $\mathbb{R}^{m}$.

Example 7.9. Consider the problem

$$
\inf \left\{\int_{0}^{1} f_{0}(t, z) d t \quad \text { s.t. } \int_{0}^{1} g_{0}(t, z) d t=a, \quad z \in \mathcal{Z}\right\},
$$

where

$$
f_{0}(t, z)=f_{0}(z)=\left\{\begin{array}{cll}
+\infty & \text { if } \quad z \leq 0 \text { or } z \geq 1, \\
\frac{1}{z(1-z)} & \text { if } \quad 0<z<1,
\end{array} \quad g_{0}(t, z)=g_{0}(z)=z .\right.
$$

$Z(t) \doteq[0,1]$ for a.e. $t \in[0,1]$. Here $W=\{0\}$.

Clearly $\psi(0)=\psi(1)=+\infty$, and since $K(a)=\emptyset$ for $a<0$ and $a>1$, we get $\psi(a)=+\infty$ for $a<0$ and $a>1$; whereas $\psi(a)<+\infty$ for $0<a<1$, since $K(a)$ is nonempty, and $z(t)=a$ for a.e. $t \in[0,1]$ is a feasible solution.

Hence, dom $\left.\psi=g\left(C_{0}\right)+W=\right] 0,1[$ is an open set.

Consider the Hamiltonian

$$
H(t, p) \doteq \sup _{\xi \in Z(t)}\left\{\left\langle p, g_{0}(t, \xi)\right\rangle-f_{0}(t, \xi)\right\}=\sup _{0<\xi<1}\left\{p \xi-\frac{1}{\xi(1-\xi)}\right\} .
$$

Note that the supremum is finite for every $p \in \mathbb{R}$, since

$$
\lim _{\xi \rightarrow 0^{+}}\left\{p \xi-\frac{1}{\xi(1-\xi)}\right\}=\lim _{\xi \rightarrow 1^{-}}\left\{p \xi-\frac{1}{\xi(1-\xi)}\right\}=-\infty
$$

and the argument of the supremum is a continuous function on $] 0,1[$. Then, $\mathcal{H}=\mathbb{R}$ and, since $g(t, z)=z$, it follows that the set $K_{0}$ is closed.

Therefore the assumptions of Theorem 7.4 are fulfilled and the problem admits an optimal solution for every $a \in] 0,1[$.

Remark 7.10. We notice that the set $I\left(K_{0}\right)$ is closed in the simplest case when $K_{0}$ is an affine set, i.e., $\forall x, y \in K_{0}, \forall \alpha \in \mathbb{R}, \alpha x+(1-\alpha) y \in K_{0}$. Then, recalling that $I: K_{0} \rightarrow \mathbb{R}^{n+1}$ is linear, $I(K)$ is an affine set in $\mathbb{R}^{m+1}$ and therefore it is closed. Clearly $K_{0}$ is affine if $f_{0}(t, \cdot)$ and $g_{0}(t, \cdot)$ are linear, for a.e. $t \in[0,1]$ and $C_{0}$ is an affine set in $L^{1}\left([0,1], \mathbb{R}^{n}\right)$.

Theorems 7.1 and 7.3 extend Theorem 3.2 and Corollary 3.1 of [6], respectively. A related existence result may be found in [1].

Acknowledgment. The authors want to express their gratitude to both referees for their helpful remarks, which have improved the paper.

\section{REFERENCES}

[1] Z. Artstein, On a variational problem, J. Math. Anal. Appl., 45 (1974), pp. 404-415.

[2] J. M. Borwein And A. S. Lewis, Partially finite convex programming, part I: Quasi relative interiors and duality theory, Math. Program. A, 57 (1992), pp. 15-48. 
[3] A. Bouras and E. Giner, Kuhn-Tucker conditions and integral functionals, J. Convex Anal., 8 (2001), pp. 533-553.

[4] F. H. Clarke, Necessary Conditions in Dynamic Optimization, Mem. Amer. Math. Soc. 816 (2005).

[5] F. H. Clarke, Functional Analysis, Calculus of Variations and Optimal Control, Springer London, Grad. Texts in Math. 264, Springer, London, 2013.

[6] G. CRAsta, Existence of minimizers for nonconvex variational problems with slow growth, J. Optim. Theory Appl., 99 (1998), pp. 381-401.

[7] F. Flores-BAZÁn And J.-P. RAYMond, A variational problem related to a continuous-time allocation process for a continuum of traders, J. Math. Anal. Appl., 261 (2001), pp. 448460.

[8] F. Hiai and H. Umegaki, Integrals, conditional expectations, and martingales of multivalued functions, J. Multivariate Analysis, 7 (1977), pp. 149-182.

[9] A. D. Ioffe And V. M. Tikhomirov, On minimization of integral functionals, Funktsional. Ana. i Prilozhen., 3 (1969), pp. 61-70 (in English).

[10] A. Jourani, Lagrangian and Hamiltonian necessary conditions for the generalized Bolza problem and applications, J. Nonlinear Convex Anal., 10 (2009), pp. 437-454.

[11] A. Jourani and L. ThiBault, Metric regularity and subdifferential calculus in Banach spaces, Set-Valued Anal., 3 (1995), pp. 87-100.

[12] B. S. Mordukhovich, Variational Analysis and Generalized Differentiation, Vol. I, Compr. Stud. Math. 330, Springer, New York, 2005.

[13] O. Olech, The Lyapunov theorem: Its extensions and applications, in Methods of Nonconvex Analysis, A. Cellina, ed., Springer Verlag, New York, 1990, pp. 86-103.

[14] J. P. Penot, Image space approach and subdifferentials of integral functionals, Optimization, 60 (2011), pp. 69-87.

[15] R. T. RockAfEllar, Conjugate Duality and Optimization, SIAM, Philadelphia, 1974.

[16] R. T. Rockafellar, Convex Analysis, Princeton University Press, Princeton, NJ, 1970.

[17] A. J. Zaslavski, Nonconvex Optimal Control and Variational Problems, Optim. Appl., 82, Springer, New York, 2013.

Copyright (c) by SIAM. Unauthorized reproduction of this article is prohibited. 